\title{
SUPERADDITIVITY OF FUNCTIONALS RELATED TO GAUSS' TYPE INEQUALITES
}

\author{
SANJA VAROŠANEC
}

\begin{abstract}
In this paper we prove superadditivity of some functionals associated with the Gauss-Winckler and the Gauss-Pólya inequalities.
\end{abstract}

\section{INTRODUCTION}

In [2] C. F. Gauss mentioned the following inequality between the second and the fourth absolute moments.

If $f$ is a non-negative and decreasing function, then

$$
\left(\int_{0}^{\infty} x^{2} f(x) d x\right)^{2} \leq \frac{5}{9} \int_{0}^{\infty} f(x) d x \int_{0}^{\infty} x^{4} f(x) d x .
$$

Until now, there are a lot of generalizations, sharpenings and improvements of inequality (1.1). One of major lines of generalization is due to A. Winckler and the other springing from a pair of results of G. Pólya.

A. Winckler, [7], gave the following result which is known as the GaussWinckler inequality in the recent literature. More about it and its history one can find in [1].

Theorem 1.1. If $f$ is a non-negative, continuous and non-increasing function on $[0, \infty)$ such that $\int_{0}^{\infty} f(x) d x=1$, then for $m \leq r$

$$
\left((m+1) \int_{0}^{\infty} x^{m} f(x) d x\right)^{\frac{1}{m}} \leq\left((r+1) \int_{0}^{\infty} x^{r} f(x) d x\right)^{\frac{1}{r}}
$$

Another generalization was done by G. Pólya and today those type of inequalities are called the Gauss-Pólya inequalites. Namely, in the book "Problems and Theorems in Analysis" (see [5, Vol I, p. 83, Vol II, p. 129] one can find the following results.

2010 Mathematics Subject Classification. 26D15.

Key words and phrases. Gauss-Winckler's inequality, Gauss-Pólya's inequalities, superadditive functional. 


\section{Theorem 1.2.}

(i) Let $f:[0, \infty) \rightarrow \mathbf{R}$ be a non-negative and decreasing function. If a and $b$ are non-negative real numbers, then

$$
\left(\int_{0}^{\infty} x^{a+b} f(x) d x\right)^{2} \leq\left(1-\left(\frac{a-b}{a+b+1}\right)^{2}\right) \int_{0}^{\infty} x^{2 a} f(x) d x \int_{0}^{\infty} x^{2 b} f(x) d x
$$

if all the integrals exist.

(ii) Let $f:[0,1] \rightarrow \mathbf{R}$ be a non-negative and increasing function. If $a$ and $b$ are non-negative real numbers, then

$$
\left(\int_{0}^{1} x^{a+b} f(x) d x\right)^{2} \geq\left(1-\left(\frac{a-b}{a+b+1}\right)^{2}\right) \int_{0}^{1} x^{2 a} f(x) d x \int_{0}^{1} x^{2 b} f(x) d x .
$$

J. Pečarić and S. Varošanec treated the above mentioned inequalities in a unified way and proved the following generalizations, [4], [6].

Theorem 1.3. Let $g:[a, b] \rightarrow \mathbf{R}$ be a non-negative increasing differentiable function and let $f:[a, b] \rightarrow \mathbf{R}$, be a non-negative function such that $x \mapsto$ $\frac{f(x)}{g^{\prime}(x)}$ is a non-decreasing function. Let $p_{i}(i=1, \ldots, n)$ be positive real numbers such that $\sum_{i=1}^{n} \frac{1}{p_{i}}=1$. If $a_{i}(i=1, \ldots, n)$ are real numbers such that $a_{i}>-\frac{1}{p_{i}}$, then

$$
\int_{a}^{b} g(x)^{a_{1}+\cdots+a_{n}} f(x) d x \geq \frac{\prod_{i=1}^{n}\left(a_{i} p_{i}+1\right)^{\frac{1}{p_{i}}}}{1+\sum_{i=1}^{n} a_{i}} \prod_{i=1}^{n}\left(\int_{a}^{b} g(x)^{a_{i} p_{i}} f(x) d x\right)^{\frac{1}{p_{i}}} .
$$

If $g(a)=0$ and if the quotient function $\frac{f}{g^{\prime}}$ is non-increasing, then the reverse inequality in (1.3) holds.

As a consequence of the above results we conclude that if $f$ and $g$ satisfy the assumptions of Theorem 1.3, then the function

$$
Q(r)=(r+1) \int_{a}^{b} g^{r}(x) f(x) d x
$$

is log-concave when $\frac{f}{g^{\prime}}$ is a non-decreasing function and the function $Q$ is log-convex when $g(a)=0$ and $\frac{f}{g^{\prime}}$ is non-increasing.

Using that property, the following generalization of the Gauss-Winckler inequality was proved in [6]:

Theorem 1.4. Let $f$ and $g$ be defined as in Theorem 1.3, $\frac{f}{g^{\prime}}$ be a nondecreasing function and $p, q, r, s$ be real numbers from the domain of definition of the function $Q$. 
If $p \leq q, r \leq s$ and $p>r, q>s$, then

$$
\left(\frac{(p+1) \int_{a}^{b} g^{p}(x) f(x) d x}{(r+1) \int_{a}^{b} g^{r}(x) f(x) d x}\right)^{\frac{1}{p-r}} \geq\left(\frac{(q+1) \int_{a}^{b} g^{q}(x) f(x) d x}{(s+1) \int_{a}^{b} g^{s}(x) f(x) d x}\right)^{\frac{1}{q-s}}
$$

If $g(a)=0$ and $\frac{f}{g^{\prime}}$ is non-increasing, then the reverse inequality holds.

Remark 1.5. In [6] the authors considered the case when $g(x)=x, f$ is non-increasing and $a=0$. In that case inequalities (1.3) and (1.4) hold with $b=\infty$ and then we get results for moments.

In the next section we investigate properties of mappings which arise from Gauss-Pólya's inequalities, while in the third section we research functional related to the Gauss-Winckler inequality (1.4). The main tool of this investigation is the Hölder type inequality which we give in the following form, [3]:

Proposition 1.6. Let $a_{i}, b_{i}, p_{i},(i=1, \ldots, n)$ be non-negative real numbers such that $\sum_{i=1}^{n} \frac{1}{p_{i}}=1$. Then

$$
a_{1}^{\frac{1}{p_{1}}} \cdots a_{n}^{\frac{1}{p_{n}}}+b_{1}^{\frac{1}{p_{1}}} \cdots b_{n}^{\frac{1}{p_{n}}} \leq \prod_{i=1}^{n}\left(a_{i}+b_{i}\right)^{\frac{1}{p_{i}}} .
$$

It is a simple consequence of weighted AM-GM inequality

$$
\begin{gathered}
\frac{a_{1}^{\frac{1}{p_{1}}} \cdots a_{n}^{\frac{1}{p_{n}}}}{\left(a_{1}+b_{1}\right)^{\frac{1}{p_{1}}} \cdots\left(a_{n}+b_{n}\right)^{\frac{1}{p_{n}}}}+\frac{b_{1}^{\frac{1}{p_{1}}} \cdots b_{n}^{\frac{1}{p_{n}}}}{\left(a_{1}+b_{1}\right)^{\frac{1}{p_{1}}} \cdots\left(a_{n}+b_{n}\right)^{\frac{1}{p_{n}}}} \\
\leq \frac{a_{1}}{p_{1}\left(a_{1}+b_{1}\right)}+\cdots+\frac{a_{n}}{p_{n}\left(a_{n}+b_{n}\right)}+\frac{b_{1}}{p_{1}\left(a_{1}+b_{1}\right)}+\cdots+\frac{b_{n}}{p_{n}\left(a_{n}+b_{n}\right)}=1 .
\end{gathered}
$$

\section{Functionals Related to the Gauss-Pólya ineQualites}

Throughout this section functions $f, g:[a, b] \rightarrow \mathbf{R}$ are non-negative, $g$ is increasing differentiable, numbers $p_{i}(i=1, \ldots, n)$ are positive reals such that $\sum_{i=1}^{n} \frac{1}{p_{i}}=1$ and $a_{i}(i=1, \ldots, n)$ are real numbers such that $a_{i}>-\frac{1}{p_{i}}$.

Let us consider the functional $\mathrm{G}$ defined as

$$
\begin{aligned}
G(f)=\prod_{i=1}^{n}\left(a_{i} p_{i}+1\right)^{\frac{1}{p_{i}}} & \prod_{i=1}^{n}\left(\int_{a}^{b} g(x)^{a_{i} p_{i}} f(x) d x\right)^{\frac{1}{p_{i}}} \\
& -\left(1+\sum_{i=1}^{n} a_{i}\right) \int_{a}^{b} g(x)^{a_{1}+\cdots+a_{n}} f(x) d x .
\end{aligned}
$$


It is obvious that $f \mapsto G(f)$ is positive homogeneous, i.e. $G(\lambda f)=\lambda G(f)$ for any $\lambda \geq 0$. As a consequence of Theorem 1.3, if $f / g^{\prime}$ is a non-decreasing function, then $G(f) \leq 0$, while if $f / g^{\prime}$ is non-increasing and $g(a)=0$, then $G(f) \geq 0$.

The following theorem gives superadditivity property of the functional $G$.

Theorem 2.1. Let $f_{1}, f_{2}, g:[a, b] \rightarrow \mathbf{R}$ be non-negative functions, $g$ increasing differentiable, numbers $p_{i}(i=1, \ldots, n)$ be positive reals such that $\sum_{i=1}^{n} \frac{1}{p_{i}}=1$ and $a_{i}(i=1, \ldots, n)$ be real numbers such that $a_{i}>-\frac{1}{p_{i}}$. Then

$$
G\left(f_{1}+f_{2}\right) \geq G\left(f_{1}\right)+G\left(f_{2}\right),
$$

i.e. $G$ is a superadditive functional.

Furthermore, if $f_{1} \geq f_{2}$ such that $\frac{f_{1}-f_{2}}{g^{\prime}}$ is non-increasing, $g(a)=0$, then

$$
G\left(f_{1}\right) \geq G\left(f_{2}\right),
$$

i.e. $G$ is non-decreasing.

Proof. Let us consider a difference $G\left(f_{1}+f_{2}\right)-G\left(f_{1}\right)-G\left(f_{2}\right)$.

$$
\begin{aligned}
G\left(f_{1}+\right. & \left.f_{2}\right)-G\left(f_{1}\right)-G\left(f_{2}\right)=\prod_{i=1}^{n}\left(a_{i} p_{i}+1\right)^{\frac{1}{p_{i}}} \prod_{i=1}^{n}\left(\int_{a}^{b} g(x)^{a_{i} p_{i}}\left(f_{1}+f_{2}\right)(x) d x\right)^{\frac{1}{p_{i}}} \\
& -\left(1+\sum_{i=1}^{n} a_{i}\right) \int_{a}^{b} g(x)^{a_{1}+\cdots+a_{n}}\left(f_{1}+f_{2}\right)(x) d x \\
& -\prod_{i=1}^{n}\left(a_{i} p_{i}+1\right)^{\frac{1}{p_{i}}} \prod_{i=1}^{n}\left(\int_{a}^{b} g(x)^{a_{i} p_{i}} f_{1}(x) d x\right)^{\frac{1}{p_{i}}} \\
& +\left(1+\sum_{i=1}^{n} a_{i}\right) \int_{a}^{b} g(x)^{a_{1}+\cdots+a_{n}} f_{1}(x) d x \\
& -\prod_{i=1}^{n}\left(a_{i} p_{i}+1\right)^{\frac{1}{p_{i}}} \prod_{i=1}^{n}\left(\int_{a}^{b} g(x)^{a_{i} p_{i}} f_{2}(x) d x\right)^{\frac{1}{p_{i}}} \\
& +\left(1+\sum_{i=1}^{n} a_{i}\right) \int_{a}^{b} g(x)^{a_{1}+\cdots+a_{n}} f_{2}(x) d x \\
& =\prod_{i=1}^{n}\left(a_{i} p_{i}+1\right)^{\frac{1}{p_{i}}}\left[\prod_{i=1}^{n}\left(\int_{a}^{b} g(x)^{a_{i} p_{i}}\left(f_{1}+f_{2}\right)(x)^{\frac{1}{p_{i}}} d x\right)^{\frac{1}{p_{i}}}-\prod_{i=1}^{n}\left(\int_{a}^{b} g(x)^{a_{i} p_{i}} f_{2}(x) d x\right)^{\frac{1}{p_{i}}}\right] . \\
& -\prod_{i=1}^{n}\left(\int_{a}^{b} g(x)^{a_{i} p_{i}} f_{1}(x) d x\right)^{b}
\end{aligned}
$$


Setting in (1.5):

$$
a_{i}=\int_{a}^{b} g(x)^{a_{i} p_{i}} f_{1}(x) d x, \quad b_{i}=\int_{a}^{b} g(x)^{a_{i} p_{i}} f_{2}(x) d x, \quad i=1,2, \ldots, n
$$

and using the Hölder inequality we have that $G\left(f_{1}+f_{2}\right)-G\left(f_{1}\right)-G\left(f_{2}\right) \geq 0$, so $G$ is superadditive.

If $f_{1} \geq f_{2}, \frac{f_{1}-f_{2}}{g^{\prime}}$ is non-increasing and $g(a)=0$, then $G\left(f_{1}-f_{2}\right) \geq 0$, so, we have

$$
\begin{aligned}
G\left(f_{1}\right) & =G\left(f_{2}+\left(f_{1}-f_{2}\right)\right) \\
& \geq G\left(f_{2}\right)+G\left(f_{1}-f_{2}\right) \geq G\left(f_{2}\right) .
\end{aligned}
$$

Corollary 2.2. Let $f_{1}, f_{2}, g$ be non-negative functions on $[a, b], g$ increasing differentiable, $g(a)=0$, numbers $p_{i}(i=1, \ldots, n)$ be positive reals such that $\sum_{i=1}^{n} \frac{1}{p_{i}}=1, a_{i}(i=1, \ldots, n)$ be real numbers such that $a_{i}>-\frac{1}{p_{i}}$ and $c, C \in \mathbf{R}$ such that $C f_{2}-f_{1}, f_{1}-c f_{2}$ are non-negative and $\frac{C f_{2}-f_{1}}{g^{\prime}}, \frac{f_{1}-c f_{2}}{g^{\prime}}$ are non-negative non-increasing functions. Then

$$
\begin{gathered}
C\left\{\prod_{i=1}^{n}\left(a_{i} p_{i}+1\right)^{\frac{1}{p_{i}}} \prod_{i=1}^{n}\left(\int_{a}^{b} g(x)^{a_{i} p_{i}} f_{2}(x) d x\right)^{\frac{1}{p_{i}}}\right. \\
\left.-\left(1+\sum_{i=1}^{n} a_{i}\right) \int_{a}^{b} g(x)^{a_{1}+\cdots+a_{n}} f_{2}(x) d x\right\} \\
\geq \prod_{i=1}^{n}\left(a_{i} p_{i}+1\right)^{\frac{1}{p_{i}}} \prod_{i=1}^{n}\left(\int_{a}^{b} g(x)^{a_{i} p_{i}} f_{1}(x) d x\right)^{\frac{1}{p_{i}}} \\
\quad-\left(1+\sum_{i=1}^{n} a_{i}\right) \int_{a}^{b} g(x)^{a_{1}+\cdots+a_{n}} f_{1}(x) d x \\
\geq\left\{\prod_{i=1}^{n}\left(a_{i} p_{i}+1\right)^{\frac{1}{p_{i}}} \prod_{i=1}^{n}\left(\int_{a}^{b} g(x)^{a_{i} p_{i}} f_{2}(x) d x\right)^{\frac{1}{p_{i}}}\right. \\
\left.-\left(1+\sum_{i=1}^{n} a_{i}\right) \int_{a}^{b} g(x)^{a_{1}+\cdots+a_{n}} f_{2}(x) d x\right\} .
\end{gathered}
$$

Proof. Using previous results we have

$$
C G\left(f_{2}\right)=G\left(C f_{2}\right)=G\left(\left(C f_{2}-f_{1}\right)+f_{1}\right) \geq G\left(C f_{2}-f_{1}\right)+G\left(f_{1}\right) \geq G\left(f_{1}\right)
$$

and

$$
G\left(f_{1}\right)=G\left(\left(f_{1}-c f_{2}\right)+c f_{2}\right) \geq G\left(f_{1}-c f_{2}\right)+G\left(c f_{2}\right) \geq G\left(c f_{2}\right)=c G\left(f_{2}\right)
$$


from which the conclusion of the corollary is established.

The following theorem contains a result about concavity of function $G \circ \phi$ where $\phi$ is concave.

Theorem 2.3. Let $\phi:[0, \infty) \rightarrow[0, \infty)$ be a concave function, $f_{1}, f_{2}, g$ be non-negative functions on $[a, b]$ such that $\left(\phi \circ\left(\alpha f_{1}+(1-\alpha) f_{2}\right)-\left[\alpha\left(\phi \circ f_{1}\right)+\right.\right.$ $\left.\left.(1-\alpha)\left(\phi \circ f_{2}\right)\right]\right) / g^{\prime}$ is non-increasing for some $\alpha \in[0,1], g(a)=0$. Then

$$
G \circ \phi \circ\left(\alpha f_{1}+(1-\alpha) f_{2}\right) \geq \alpha\left(G \circ \phi \circ f_{1}\right)+(1-\alpha)\left(G \circ \phi \circ f_{2}\right) .
$$

Proof. For any $x \in[a, b]$ we have

$$
\begin{aligned}
\left(\phi \circ\left(\alpha f_{1}+(1-\alpha) f_{2}\right)\right)(x) & =\phi\left(\alpha f_{1}(x)+(1-\alpha) f_{2}(x)\right) \\
& \geq \alpha \phi\left(f_{1}(x)\right)+(1-\alpha) \phi\left(f_{2}(x)\right) \\
& =\left(\alpha\left(\phi \circ f_{1}\right)+(1-\alpha)\left(\phi \circ f_{2}\right)\right)(x),
\end{aligned}
$$

where a concavity of function $\phi$ is used. So, we have $\phi \circ\left(\alpha f_{1}+(1-\alpha) f_{2}\right) \geq$ $\alpha\left(\phi \circ f_{1}\right)+(1-\alpha)\left(\phi \circ f_{2}\right)$. Using properties of $G$ and the above-proved inequality we have

$$
\begin{aligned}
& G\left(\phi \circ\left(\alpha f_{1}+(1-\alpha) f_{2}\right)\right) \geq G\left(\alpha\left(\phi \circ f_{1}\right)+(1-\alpha)\left(\phi \circ f_{2}\right)\right) \\
& \quad \geq G\left(\alpha\left(\phi \circ f_{1}\right)\right)+G\left((1-\alpha)\left(\phi \circ f_{2}\right)\right)=\alpha G\left(\phi \circ f_{1}\right)+(1-\alpha) G\left(\phi \circ f_{2}\right)
\end{aligned}
$$

and the proof is established.

Remark 2.4. Let us consider a case when $g(x)=x, a=0, b=\infty$ and $f$ is non-increasing as it is mentioned in Remark 1.5. Let us denote by $\mu_{r}(f)$ a moment of the order $r$ i.e.

$$
\mu_{r}(f)=\int_{0}^{\infty} x^{r} f(x) d x
$$

Then the functional $G$ has a form

$$
G(f)=\prod_{i=1}^{n}\left(a_{i} p_{i}+1\right)^{\frac{1}{p_{i}}} \prod_{i=1}^{n} \mu_{a_{i} p_{i}}^{\frac{1}{p_{i}}}(f)-\left(1+\sum_{i=1}^{n} a_{i}\right) \mu_{a_{1}+\cdots+a_{n}}(f)
$$

and $G$ is superadditive. Also, if $f_{1} \geq f_{2}$ such that $f_{1}-f_{2}$ is non-increasing, then $G\left(f_{1}\right) \geq G\left(f_{2}\right)$.

\section{Functionals Related to the Gauss-Winckler inequality}

Putting in (1.4) $r=s=0$ we get the Gauss-Winckler inequality for $f / g^{\prime}$ non-decreasing function:

$$
\left(\frac{(p+1) \int_{a}^{b} g^{p}(x) f(x) d x}{\int_{a}^{b} f(x) d x}\right)^{\frac{1}{p}} \geq\left(\frac{(q+1) \int_{a}^{b} g^{q}(x) f(x) d x}{\int_{a}^{b} f(x) d x}\right)^{\frac{1}{q}}
$$


where $0<p \leq q$. If $f / g^{\prime}$ is non-increasing and $g(a)=0$, then the reversed inequality holds.

Let us consider a functional $W$ defined as

$$
\begin{aligned}
W(f)=\left(\int_{a}^{b} f(x) d x\right)^{1-\frac{p}{q}}\left((q+1) \int_{a}^{b} g^{q}(x)\right. & f(x) d x)^{\frac{p}{q}} \\
& -(p+1) \int_{a}^{b} g^{p}(x) f(x) d x .
\end{aligned}
$$

The following theorem gives superadditivity and monotonicity of the functional $W$.

Theorem 3.1. Let $f_{1}, f_{2}, g:[a, b] \rightarrow \mathbf{R}$ be non-negative functions, $g$ increasing differentiable, numbers $p, q$ be positive real such that $p \leq q$. Then

$$
W\left(f_{1}+f_{2}\right) \geq W\left(f_{1}\right)+W\left(f_{2}\right) .
$$

Additionally, if $f_{1} \geq f_{2}$ such that $\frac{f_{1}-f_{2}}{g^{\prime}}$ is non-increasing, $g(a)=0$, then

$$
W\left(f_{1}\right) \geq W\left(f_{2}\right) .
$$

Proof. Let us transform $W\left(f_{1}+f_{2}\right)-W\left(f_{1}\right)-W\left(f_{2}\right)$.

$$
\begin{aligned}
& W\left(f_{1}+f_{2}\right)-W\left(f_{1}\right)-W\left(f_{2}\right) \\
& =\left(\int_{a}^{b}\left(f_{1}+f_{2}\right)(x) d x\right)^{1-\frac{p}{q}}\left((q+1) \int_{a}^{b} g^{q}(x)\left(f_{1}+f_{2}\right)(x) d x\right)^{\frac{p}{q}} \\
& -(p+1) \int_{a}^{b} g^{p}(x)\left(f_{1}+f_{2}\right)(x) d x \\
& -\left(\int_{a}^{b} f_{1}(x) d x\right)^{1-\frac{p}{q}}\left((q+1) \int_{a}^{b} g^{q}(x) f_{1}(x) d x\right)^{\frac{p}{q}} \\
& +(p+1) \int_{a}^{b} g^{p}(x) f_{1}(x) d x-\left(\int_{a}^{b} f_{2}(x) d x\right)^{1-\frac{p}{q}}\left((q+1) \int_{a}^{b} g^{q}(x) f_{2}(x) d x\right)^{\frac{p}{q}} \\
& +(p+1) \int_{a}^{b} g^{p}(x) f_{2}(x) d x \\
& =\left(\int_{a}^{b}\left(f_{1}+f_{2}\right)(x) d x\right)^{1-\frac{p}{q}}\left((q+1) \int_{a}^{b} g^{q}(x)\left(f_{1}+f_{2}\right)(x) d x\right)^{\frac{p}{q}} \\
& -\left(\int_{a}^{b} f_{1}(x) d x\right)^{1-\frac{p}{q}}\left((q+1) \int_{a}^{b} g^{q}(x) f_{1}(x) d x\right)^{\frac{p}{q}} \\
& -\left(\int_{a}^{b} f_{2}(x) d x\right)^{1-\frac{p}{q}}\left((q+1) \int_{a}^{b} g^{q}(x) f_{2}(x) d x\right)^{\frac{p}{q}} \geq 0
\end{aligned}
$$


where in the last inequality we use the Hölder inequality with

$$
\begin{gathered}
n=2, \frac{1}{p_{1}}=1-\frac{p}{q}>0, \frac{1}{p_{2}}=\frac{p}{q}>0, \quad a_{1}=\int_{a}^{b} f_{1}(x) d x, \quad b_{1}=\int_{a}^{b} f_{2}(x) d x, \\
a_{2}=(q+1) \int_{a}^{b} g^{q}(x) f_{1}(x) d x, \quad b_{2}=(q+1) \int_{a}^{b} g^{q}(x) f_{2}(x) d x .
\end{gathered}
$$

So, superadditivity of the functional $\mathrm{W}$ is established.

If $\frac{f_{1}-f_{2}}{g^{\prime}}$ is non-increasing, $g(a)=0$, then from Theorem 1.4 we obtain $W\left(f_{1}-f_{2}\right) \geq 0$ and

$$
W\left(f_{1}\right)=W\left(f_{2}+\left(f_{1}-f_{2}\right)\right) \geq W\left(f_{2}\right)+W\left(f_{1}-f_{2}\right) \geq W\left(f_{2}\right) .
$$

Remark 3.2. Let us consider a case when $g(x)=x, a=0, b=\infty$ and $f$ is non-increasing as it is mentioned in Remark 1.5. Now the functional $W$ has the form

$$
W(f)=(q+1)^{\frac{p}{q}}\left(\mu_{0}(f)\right)^{1-\frac{p}{q}} \mu_{q}^{\frac{p}{q}}(f)-(p+1) \mu_{p}(f)
$$

and $W$ is superadditive. Also, if $f_{1} \geq f_{2}$ such that $f_{1}-f_{2}$ is non-increasing, then $W\left(f_{1}\right) \geq W\left(f_{2}\right)$.

The following result is an interesting inequality for the Beta function.

Corollary 3.3. Let $0<p \leq q, y_{1}, y_{2}>-1$. Then

$$
\begin{aligned}
& \left(\frac{1}{y_{1}+1}+\frac{1}{y_{2}+1}\right)^{1-\frac{p}{q}}\left[\beta\left(q+1, y_{1}+1\right)+\beta\left(q+1, y_{2}+1\right)\right]^{\frac{p}{q}} \\
\geq & \left(\frac{1}{y_{1}+1}\right)^{1-\frac{p}{q}} \beta^{\frac{p}{q}}\left(q+1, y_{1}+1\right)+\left(\frac{1}{y_{2}+1}\right)^{1-\frac{p}{q}} \beta^{\frac{p}{q}}\left(q+1, y_{2}+1\right)
\end{aligned}
$$

where $\beta$ is the Beta function defined as $\beta(x+1, y+1)=\int_{0}^{1} t^{x}(1-t)^{y} d t$.

Proof. It is a consequence of the previous theorem with $[a, b]=[0,1], f_{i}(t)=$ $(1-t)^{y_{i}}, i=1,2, g(x)=x$.

\section{REFERENCES}

[1] P. R. Beesack, Inequalities for absolute moments of a distribution: From Laplace to Von Mise, J. Math. Anal. Appl., 98 (1984), 435-457.

[2] C. F. Gauss, Theoria combinationis observationum, 1821.

[3] D. S. Mitrinović, J. E. Pečarić and A. M. Fink, Classical and New Inequalities in Analysis. Dordrecht, Kluwer Acad. Publishers, 1993.

[4] J. Pečarić and S. Varošanec, Remarks on Gauss-Winckler's and Stolarsky's inequalities, Utilitas Math., 48 (1995), 233-241. 
[5] G. Pólya and G. Szegö, Aufgaben und Lehrsätze aus der Analysis, Vol I and II. Berlin, Springer Verlag, 1925.

[6] S. Varošanec and J. Pečarić, Gauss' and related inequalites, Z. Anal. Anwendungen, 14 (1995), 175-183.

[7] A. Winckler, Allgemeine Sätze zur Theorie der unregelmäßigen Beobachtungsfehler, Sitzungsber. Akad. Wiss. Wien, Math.-Natur. Kl. Zweite Abt., 53 (1866), 6-41.

(Received: May 22, 2013)

(Revised: July 19, 2013)

Department of Mathematics

University of Zagreb

10000 Zagreb, Bijenička c. 30

Croatia

varosans@math.hr 